\title{
Decomposition of Differential Polynomials with Constant Coefficients:
}

\author{
Xiao-Shan Gao and Mingbo Zhang \\ Key Laboratory of Mathematics Mechanization \\ Institute of Systems Science, AMSS, \\ Academia Sinica, Beijing 100080, China \\ (xgao,mzhang)@mmrc.iss.ac.cn
}

\begin{abstract}
In this paper, we present an algorithm to decompose differential polynomials in one variable and with rational number as coefficients. Besides arithmetic operations, the algorithm needs only factorization of multi-variable polynomials and solution of linear equation systems. Experimental results show that our method is quite efficient.
\end{abstract}

\section{Categories and Subject Descriptors}

I.1.2 [SYMBOLIC AND ALGEBRAIC MANIPULATION ]: Algorithms - Algebraic algorithms

\section{General Terms}

Algorithm

\section{Keywords}

differential polynomial, decomposition, pseudo linear, differential degree

\section{INTRODUCTION AND PRELIMINARIES}

The study on functional decomposition started with the decomposition of univariate polynomials (pols). The first decomposition algorithms were presented by Barton-Zippel [3] and Alagar-Tanh [1]. This was followed by the work of Kozen and Landau [16] who proposed a pol time decomposition algorithm. Similar algorithms were given by Gutierrez [12]. Gathen proposed an algorithm of better complexity and a parallel algorithm in [9]. In [29], Zippel presented a pol time algorithm to decompose a given univariate rational function over an arbitrary field.

Decomposing linear ordinary differential equations (LODEs) was first discussed by Singer in [19]. The algorithms to

\footnotetext{
Partially supported by a National Key Basic Research Project of China and by a USA NSF grant CCR-0201253.
}

Permission to make digital or hard copies of all or part of this work for personal or classroom use is granted without fee provided that copies are not made or distributed for profit or commercial advantage and that copies bear this notice and the full citation on the first page. To copy otherwise, to republish, to post on servers or to redistribute to lists, requires prior specific permission and/or a fee.

ISSAC'04, July 4-7, 2004, Santander, Spain.

Copyright 2004 ACM 1-58113-827-X/04/0007 ...\$5.00. decompose LODEs proposed by Bronstein, Petkovšek, and Schwarz $[4,5,18]$ were based on the classical work of Beke. Another approach based on the eigenring of the LODE was proposed by van der Put and Singer [28]. In [26, 27], van Hoeij proposed an algorithm to decompose an LODE with rational functions as well as power series coefficients, which was extended to decompose LODEs over an exponential extension of a base field by Fredet [7]. In [23], Tsarev presented an algorithm for complete enumeration of all factorizations of an LODE. In $[2,6]$, Barkatou-Pflügel and Cluzeau proposed decomposition algorithms for LODE systems. In [10], Giesbrecht and Zhang presented methods to find decomposition for the more general Ore pols. In [11], Grigor'ev gave an algorithm to decompose LODEs and showed that the worst case complexity of the algorithm is exponential. Applications of the decomposition of LODEs to compute closed form solutions and to determine the Galois group were discussed by Singer and Ulmer [19, 21]. In [13], Li et al gave the first algorithm to decompose systems of linear PDEs with finite-dimensional solution spaces.

The problem of decomposing non-linear differential polynomials ( $d$-pols) was discussed in the classic work by Königsberger [15]. Methods for some special classes of $d$-pols were given in $[22,25]$. In [24], Tsarev considered $d$-pols of the form $y_{n}-R\left(x, y, \ldots, y_{n-1}\right)$ and gave a decision procedure for the existence of factorization. As far as we know, there exist no effective methods to decompose non-linear $d$-pols.

In this paper, we give an algorithm for decomposing $d$-pols in one variable and with constant coefficients. The algorithm to find a decomposition $f=g \circ h$ for a given $f$ consists of three main steps. First, we try to find a decomposition where $g$ is a univariate pol. Second, the problem is reduced to the case where $g$ is pseudo linear. Third, the pseudo linear case is solved.

Besides arithmetic operations, the algorithm needs only factorization of multi-variable pols and solution of linear equation systems, both of which have pol-time algorithms. Our algorithm is exponential in the worst case due to the need to select all possible left decomposition factors of an LODE. We implement our algorithm in Maple and experimental results show that our method is quite efficient in handling large d-pols. We use examples to show that the algorithm can be used to simplify the solution of non-linear differential equations. We also discuss shortly how to extend the algorithm in the paper to the case when the coefficients of the $d$-pols are rational functions. 


\section{PRELIMINARY RESULTS}

Let $\mathbb{Q}$ be the field of rational numbers, $\mathbb{Q}(t)$ the field of rational functions in $t, x$ a differential indeterminate, $\mathbb{Q}(t)\{x\}$ the ordinary $d$-pol ring over $\mathbb{Q}(t)$ with differentiation $\frac{\partial}{\partial t}[14]$. An element in $\mathbb{Q}(t)\{x\}$ is called a $d$-pol. We denote by $x_{i}$ the $i$-th derivative of $x$. For a $d$-pol $f$, let $x_{o}$ be the highest derivative appearing in $f$. Then $o$ is called the order of $f$ and is denoted by $o_{f}$. Let $o_{f}$ be the order of $f, d_{f}$ the degree of $f$ in $x_{o_{f}}, i_{f}$ the coefficient of $x_{o_{f}}^{d_{f}}$ in $f, s_{f}=\frac{\partial f}{\partial x_{o_{f}}}$. $d_{f}, i_{f}, s_{f}$ are called the degree, initial, and separant of $f$ respectively.

For a $d$-pol $f$ and a non-negative integer $k$, let $f_{(k)}$ be the $k$-th derivative of $f$. Then for $k>0$, we have

$$
f_{(k)}=s_{f} x_{o_{f}+k}+R_{f}
$$

where $R_{f}$ is a $d$-pol of lower order in $x$ than $o_{f}+k$.

A monomial in $\mathbb{Q}(t)\{x\}$ is always arranged in the form $a \prod_{i=1}^{r} x_{\beta_{i}}^{\alpha_{i}}$ where $a \in \mathbb{Q}(t), \alpha_{i} \in \mathbb{N}^{+}, \beta_{1}>\ldots>\beta_{r} \geq 0$. The number $\sum_{i=1}^{r} \alpha_{i}$ is called the total degree and $\sum_{i=1}^{r} \alpha_{i} \cdot \beta_{i}$ is called the differential degree of the monomial. The largest total degree and differential degree for all monomials in a $d$-pol $f$ is called the total degree and differential degree of $f$, denoted by $\operatorname{tdeg}(f)$ and $\operatorname{ddeg}(f)$ respectively.

Let $q$ be a $d$-pol. If the total (differential) degrees of the monomials in $q$ are all equal, $q$ is called total (differential) degree homogeneous. If $\operatorname{tdeg}(q)=k$ and $q$ is total degree homogeneous, $q$ is called $k$-total degree homogeneous. Furthermore, if $k=1$ we say that $q$ is linear.

We may define a rank between two monomials according to the pure lexicographical order induced by the variable order $x<x_{1}<x_{2}<\ldots$. In a $d$-pol $p$, the term with the highest rank is called the leading term of $p$.

In the rest of this paper, we will assume that $d$-pols are with coefficients in $\mathbb{Q}$ unless mentioned otherwise. Let $g, h \in$ $\mathbb{Q}\{x\}$, we use $g \circ h$ to denote the composition of two $d$-pols $g$ and $h$, which is obtained by substituting $x_{i}$ in $g$ by $h_{(i)}$. If $f=g \circ h, g, h$ are called the left and right decomposition factor of $f$ respectively. A decomposition $f=g \circ h$ is called non-trivial, if both $g$ and $h$ are not in the form $a x+b(a, b \in$ Q).

LEMMA 1. The composition operation is associate: $f \circ(g \circ$ $h)=(f \circ g) \circ h$.

Proof. Omitted. See the preprint [8].

For $c \in \mathbb{Q}$, we have $f=g \circ h=[g \circ(x+c)] \circ[(x-c) \circ h]$. Then we may always assume that $h$ has no term in $\mathbb{Q}$. In this case, the constant term of $f$ is the same as that of $g$. So we may further assume that $f$ and $g$ have no constant terms.

In what follows, we assume that $f$ is a d-pol in $\mathbb{Q}\{x\}$ of positive order and has no term in $\mathbb{Q}$. We will find a nontrivial decomposition $f=g \circ h$. We may write $f, g, h$ as follows.

$$
f=i_{f} x_{o_{f}}^{d_{f}}+f_{1}, g=i_{g} x_{o_{g}}^{d_{g}}+g_{1}, h=i_{h} x_{o_{h}}^{d_{h}}+h_{1}
$$

where $f_{1}, g_{1}, h_{1}$ are of lower degree in $x_{o_{f}}, x_{o_{g}}, x_{o_{h}}$ than $d_{f}, d_{g}, d_{h}$ respectively.

LEMMA 2. If $f=g \circ h$, we have

$$
o_{f}=o_{g}+o_{h}, f_{(1)}=g_{(1)} \circ h, s_{f}=\left(s_{g} \circ h\right) \cdot s_{h} .
$$

Proof. Omitted. See the preprint [8].

LEMmA 3. If $f=g \circ h$ and $g$ is a univariate pol, we have

$$
\begin{aligned}
& o_{f}=o_{h}, d_{f}=d_{g} d_{h}, i_{f}=\left(i_{g} \circ h\right) i_{h}^{d_{g}} \\
& f_{(1)}=\left(s_{g} \circ h\right) h_{(1)} .
\end{aligned}
$$

Proof. Omitted. See the preprint [8].

LEMmA 4. If $f=g \circ h$ and $o_{g}>0$, we have

$$
d_{f}=d_{g}, i_{f}=\left(i_{g} \circ h\right) \cdot s_{h}^{d_{f}}
$$

Proof. Omitted. See the preprint [8].

Lemma 5. $q, u, v$ are d-pols, $q=u \circ v$. Let $d=\operatorname{tdeg}(q), d_{1}=$ $\operatorname{tdeg}(u), d_{2}=\operatorname{tdeg}(v)$, then $d=d_{1} \cdot d_{2}$. Furthermore, if $Q_{d}, U_{d_{1}}, V_{d_{2}}$ are the sums of monomials in $q, u, v$ with total degree $d, d_{1}, d_{2}$ respectively, then $Q_{d}=U_{d_{1}} \circ V_{d_{2}}$.

Proof. Omitted. See the preprint [8].

\section{POLYNOMIAL DECOMPOSITION}

We first consider a special case $o_{g}=0$, called polynomial decomposition. This can be treated as a composition of a univariate pol and a multi-variable pol in $\mathbb{Q}\left[x, x_{1}, \ldots, x_{o_{f}}\right]$, which is considered in [9]. In what follows, we give a simple algorithm based on differentiation, which is quite efficient as shown by our experimental results.

Before presenting the algorithm, we first give two basic algorithms which will also be used in other sections.

Algorithm 1. Input: d-pols $f, h \in \mathbb{Q}\{x\}$.

Output: a d-pol $g \in \mathbb{Q}\{x\}$ such that $f=g \circ h$ if such a $g$ exists.

S1 If $f \in \mathbb{Q}$, return $g=f$.

S2 Write $f$ and $h$ as (2). If $o_{f}<o_{h}$, g does not exist. The algorithm terminates.

S3 If $o_{f}=o_{h}$, from (3), $o_{g}=0$. From (4), $d_{g}=d_{f} / d_{h}$. If $d_{g}$ is not an integer, $g$ does not exist and the algorithm terminates. Otherwise, let $t=\frac{i_{f}}{i_{h}^{d g}}$. From (4), $t$ should be $i_{g} \circ h$. Go to $\mathbf{S 5}$.

S4 If $o_{f}>o_{h}$, from (3) and (6) the order $o_{g}$ and leading degree $d_{g}$ for $g$ can be computed as follows: $o_{g}=o_{f}-$ $o_{h}, d_{g}=d_{f}$. Let $t=\frac{i_{f}}{s_{h}}$. From (6), $t$ should be $i_{g} \circ h$.

S5 If $t$ is not a $d$-pol in $\mathbb{Q}\{x\}, g$ does not exist. The algorithm terminates. Otherwise, let $f_{1}=f-t \cdot h_{\left(o_{g}\right)}^{d_{g}}$. Then $f_{1}=g \circ h-\left(i_{g} \circ h\right)\left(x_{o_{g}}^{d_{g}} \circ h\right)=\left(g-i_{g} x_{o_{g}}^{d_{g}}\right) \circ h$, which also has a right decomposition factor $h$. Call Algorithm 1 with $f_{1}, h$ and $t, h$ as inputs. Let the outputs be $g_{1}$ and $i_{g}$. If $i_{g}$ and $g_{1}$ exist, return $g=i_{g} x_{o g}^{d_{g}}+g_{1}$. Otherwise, $g$ does not exist.

EXAMPLE 1. Let $f=2 x_{2}\left(x_{2}^{2}+x_{1}\right) x_{3}+x_{2}^{4}+x_{2}^{3}+2 x_{1} x_{2}^{2}+$ $x_{1} x_{2}+x_{1}^{2}, h=x_{2}^{2}+x_{1}$. Since $o_{f}=3>o_{h}=2$, we will execute $S_{4}$, where $d_{g}=d_{f}=1, o_{g}=o_{f}-o_{h}=1, t=\frac{i_{f}}{s_{h}}=$ $\frac{2 x_{2}\left(x_{2}^{2}+x_{1}\right)}{2 x_{2}}=x_{2}^{2}+x_{1}=i_{g} \circ h$. In S5, $f_{1}=f-t \cdot h_{(1)}=$ $x_{2}^{4}+2 x_{1} x_{2}^{2}+x_{1}^{2}$. Execute Algorithm 1 with input $t, h$ we 
have $i_{g}=x$. Executing Algorithm 1 with input $f_{1}, h$ we have $g_{1}=x^{2}$. So $h$ is a right decomposition factor of $f$ and the corresponding left decomposition factor is $g=i_{g} x_{o_{g}}^{d_{g}}+g_{1}=$ $x x_{1}+x^{2}$.

Algorithm 2. Input: a d-pol $p \in \mathbb{Q}\{x\}$.

Output: a d-pol $q \in \mathbb{Q}\{x\}$ such that $q_{(1)}=p$ if such a $q$ exists.

S1 Set $q=0$.

S2 If $p \notin \mathbb{Q}$ then go to the next step. If $p=0$, return $q$. Otherwise, $q$ does not exist. The algorithm terminates.

S3 Let the leading term of $p$ under the lexical order be $t=c_{p} \prod_{i=1}^{r} x_{\beta_{i}}^{\alpha_{i}}$ where $\beta_{1}>\ldots>\beta_{r} \geq 0$, and $c_{p} \in \mathbb{Q}$.

S4 If $\alpha_{1} \neq 1$ then $q$ does not exist and terminate.

S5 If $r=1$ or $\beta_{1}>\beta_{2}+1$, let

$$
s=c_{p} x_{\beta_{1}-1} \prod_{i=2}^{r} x_{\beta_{i}}^{\alpha_{i}} .
$$

Otherwise, let

$$
s=\frac{c_{p}}{\alpha_{2}+1} x_{\beta_{1}-1}^{\alpha_{2}+1} \prod_{i=3}^{r} x_{\beta_{i}}^{\alpha_{i}} .
$$

If $t>r$ in a product $\prod_{i=t}^{r}$, the value of the product is defined to be 1 .

S6 Set $q=q+s, p=p-s_{(1)}$. Go to $\mathrm{S} 2$.

To see that Algorithm 2 is correct, let the leading term of $q$ be $t=c_{q} \prod_{i=1}^{r} x_{\tau_{i}}^{\gamma_{i}}$ where $\tau_{1}>\ldots>\tau_{r} \geq 0$. The case $r=1$ is obvious. For $r>1$, the leading term of $q_{(1)}$ is

$$
l=c_{q} \gamma_{1} x_{\tau_{1}+1} x_{\tau_{1}}^{\gamma_{1}-1} \prod_{i=2}^{r} x_{\tau_{i}}^{\gamma_{i}} .
$$

Since $q_{(1)}=p, l$ should be the leading term of $p$. Then the degree of $p$ should be one, which implies the correctness of S4. If $\gamma_{1}>1, x_{\tau_{1}}$ appears in $l$, which implies the first case in S5. If $\gamma_{1}=1, x_{\tau_{1}}$ does not appear in $l$, which implies the second case in S5.

Algorithm 3. Input: a d-pol $f$.

Output: a univariate pol $g$ and a d-pol h such that $f=g \circ h$ if such $g$ and $h$ exist.

S1 If $f=g \circ h$ is a pol decomposition of $f$, from (5) $h_{(1)}$ is a proper factor of $f_{(1)}$ of order $o_{f}+1$ and with no constant terms. Let $S$ be the set of such primitive factors of $f_{(1)}$.

S2 For each $p \in S$, do S3 and S4.

S3 Use Algorithm 2 to find an $h \in \mathbb{Q}\{x\}$ such that $h_{(1)}=p$. If such an $h$ does not exist, goto $\mathrm{S} 2$.

S4 Use Algorithm 1 to find a $g \in \mathbb{Q}[x]$ such that $f=g \circ h$. If such a $g$ exists, return $g$ and $h$; else, goto S2.
ExAmple 2. Let $f=x^{2} x_{2}^{4}+\left(2 x x_{1}+x\right) x_{2}^{2}+x_{1}^{2}+x_{1}$. We have

$$
f_{(1)}=x_{2}\left(2 x x_{2}^{2}+2 x_{1}+1\right)\left(2 x x_{3}+x_{1} x_{2}+1\right)
$$

The set of proper factors for $f_{(1)}$ of order $o_{f}+1=3$ and containing no terms in $\mathbb{Q}$ is $S=\left\{p: p=x_{2}\left(2 x_{3} x+x_{2} x_{1}+\right.\right.$ 1)\}. With Algorithm 2 we find $h=x x_{2}^{2}+x_{1}$ such that $h_{(1)}=$ $p$. With Algorithm 1 we find $g=x^{2}+x$ such that $f=g \circ h$. So we get the pol decomposition $f=\left(x^{2}+x\right) \circ\left(x x_{2}^{2}+x_{1}\right)$.

\section{REDUCTION TO PSEUDO LINEAR CASE}

A $d$-pol $p$ in $\mathbb{Q}\{x\}$ is called pseudo linear if $p$ is of the form

$$
p=c x_{o_{p}}+p_{1}
$$

where $c \in \mathbb{Q}$ and $p_{1}$ is of lower order than $o_{p}$.

In what follows, we will assume that $o_{g}>0$ and compute the decomposition $f=g \circ h$. Since $o_{g}>0$, by (6), we have $d_{f}=d_{g}$. Let $d=d_{f}=d_{g}$ and

$$
\begin{aligned}
f & =f_{d} x_{o_{f}}^{d}+f_{d-1} x_{o_{f}}^{d-1}+\ldots+f_{1} x_{o_{f}}+f_{0} \\
g & =g_{d} x_{o_{g}}^{d}+g_{d-1} x_{o_{g}}^{d-1}+\ldots+g_{1} x_{o_{g}}+g_{0}
\end{aligned}
$$

By (1), $x_{o_{g}} \circ h=s_{h} x_{o_{g}+o_{h}}+R_{h}=s_{h} x_{o_{f}}+R_{h}\left(o_{R_{h}}<o_{g}+o_{h}\right)$. We have $f=\left(g_{d} \circ h\right)\left(s_{h} x_{o_{f}}+R_{h}\right)^{d}+\ldots+g_{0} \circ h$. Let $a_{i}=g_{i} \circ h$, comparing the coefficients of $x_{o_{f}}^{i}(0 \leq i \leq d)$, we have

$$
\begin{aligned}
f_{d}= & s_{h}^{d}\left(g_{d} \circ h\right) \\
& \ldots \\
f_{k}= & s_{h}^{k}\left(\left(\begin{array}{l}
d \\
k
\end{array}\right) a_{d} R_{h}^{d-k}+\left(\begin{array}{c}
d-1 \\
k
\end{array}\right) a_{d-1} R_{h}^{d-k-1}+\ldots+a_{k}\right) \\
& \ldots \\
f_{0}= & a_{d} R_{h}^{d}+a_{d-1} R_{h}^{d-1}+\ldots+a_{1} R_{h}+a_{0}
\end{aligned}
$$

Let $T=\{H: H=1$ or $H$ is a primitive $d$-pol with integer coefficients and $H^{i}$ is the factor of $\left.f_{i}(1 \leq i \leq d)\right\}$. From (8), we can assume that $s_{h}$ is in $T$. The basic idea of our algorithm is as follows: for each $H$ in $T$, we will examine whether there exists a decomposition $f=g \circ h$ such that $s_{h}=H$.

LEMMA 6. Let $f=g \circ h\left(o_{g}>0\right), d=d_{f}\left(=d_{g}\right) . s^{(k)}(f)=$ $\frac{\partial^{k} f}{\partial^{k} x_{o f}}, s^{(k)}(g)=\frac{\partial^{k} g}{\partial^{k} x_{o g}}(1 \leq k \leq d)$. Then we have $s^{(i)}(f) / s_{h}^{i}=$ $s^{(i)}(g) \circ h(1 \leq i \leq d)$.

Proof. By the third equation in (3), we have $s^{(1)}(f) / s_{h}=$ $s_{f} / s_{h}=s_{g} \circ h$. This proves the case for $i=1$. Suppose that the lemma is valid for $i=k$, that is, we have $s^{(k)}(f) / s_{h}^{k}=$ $s^{(k)}(g) \circ h$. Using (3) to the formula above again, we have $s^{(k+1)}(f) / s_{h}^{k+1}=\frac{\partial\left(s^{(k)}(f) / s_{h}^{k}\right)}{\partial x_{o}} s_{h}^{-1}=\left(\frac{\partial\left(s^{(k)}(g)\right)}{\partial x_{o g}} \circ h\right) s_{h} s_{h}^{-1}=$ $s^{(k+1)}(g) \circ h$. We may move $s_{h}$ out from the scope of the partial differentiation, because $o_{s_{h}}<o_{f}=o_{s_{f}}$ when $d>1$.

In Lemma 6 , setting $i=d-1$, we have $s^{d-1}(f) / s_{h}^{d-1}=$ $s^{d-1}(g) \circ h$. By direct computation, $s^{d-1}(f)=\frac{\partial^{d-1} f}{\partial^{d-1} x_{o f}}=$ $d ! f_{d} x_{o_{f}}+(d-1) ! f_{d-1}, s^{d-1}(g)=d ! g_{d} x_{o_{g}}+(d-1) ! g_{d-1}$. Substituting them into $s^{d-1}(f) / s_{h}^{d-1}=s^{d-1}(g) \circ h$, we have

$$
\left(f_{d} x_{o_{f}}+\frac{1}{d} f_{d-1}\right) / s_{h}^{d-1}=\left(g_{d} x_{o_{g}}+\frac{1}{d} g_{d-1}\right) \circ h
$$


We consider two cases .

Case 1. If $\frac{f_{d}}{s_{h}^{d}} \notin \mathbb{Q}$, then from (8) $h$ is a right decomposition factor of $\frac{f_{d}}{s_{h}^{d}}$. Then we can repeat the procedure for $\frac{f_{d}}{s_{h}^{d}}$. Notice that the left decomposition factor of $\frac{f_{d}}{s_{h}^{d}}$ could be a pol, but the analysis above assumes that $o_{g}>0$. So we will use Algorithm 3 to test whether $\frac{f_{d}}{s_{h}^{d}}$ has a pol decomposition before the recursion.

Case 2. If $\frac{f_{d}}{s_{h}^{d}}=a \in \mathbb{Q}$, then by (9) and (1) we have

$$
\begin{aligned}
s_{h} x_{o_{f}}+\frac{1}{a d} \cdot \frac{f_{d-1}}{s_{h}^{d-1}} & =\left(\frac{g_{d}}{a} x_{o_{g}}+\frac{1}{a d} g_{d-1}\right) \circ h \\
& =\frac{g_{d}}{a}\left(s_{h} x_{o_{f}}+R_{h}\right)+\frac{1}{a d} g_{d-1} \circ h .
\end{aligned}
$$

Compare the coefficients of $x_{o_{f}}$, we have $s_{h}=\left(\frac{g_{d}}{a} \circ h\right) \cdot s_{h}$, which implies $\frac{g_{d}}{a}=1$. Let $w=\frac{1}{a d} \cdot \frac{f_{d-1}}{s_{h}^{d-1}}, g_{1}=\frac{1}{a d} g_{d-1}$. Then

$$
p=s_{h} x_{o_{f}}+w=\left(x_{o_{g}}+g_{1}\right) \circ h
$$

The left decomposition factor of $p$ is pseudo linear.

The following algorithm is based on the analysis above.

Algorithm 4. Input: d-pols $f, H$.

Output: $d$-pols $g$ and $h$ such that $f=g \circ h$, or a d-pol $p$ such that if $h$ is a right decomposition factor of $f$ with separant $H$, then $h$ is a right decomposition factor of $p$ and the corresponding left decomposition factor of $p$ w.r.t.h is pseudo linear, or return the empty set which denotes that $f$ has no right decomposition factor with separant $H$.

S1 $t=f$.

S2 Let $d=d_{t}$. Write $t$ as the form $t=t_{d} x_{o_{t}}^{d}+\ldots+$ $t_{1} x_{o_{t}}+t_{0}$. If $o_{t}<o_{H}$, then output the empty set and terminate the algorithm; otherwise, we will try to find a decomposition $t=r \circ h$ such that $s_{h}=H$.

S3 If $a=\frac{s_{t}}{H} \in \mathbb{Q}$, by (3), $s_{r} \circ h=s_{t} / s_{h}=s_{t} / H=a$, so $s_{r}=a$ and $r$ is pseudo linear. Notice that the order of $r$ could be 0 , which means $t$ is a possible right decomposition factor of $f$. Call Algorithm 1 with $f$ and $t$ as input. If we find a $g$ such that $f=g \circ t$, then output $g$ and $t$; else, output $p=t$. Terminate the algorithm.

$\mathbf{S} 4$ Execute Algorithm 3 with input $t$. If we find $r, h$ such that $t=r \circ h$ and $\frac{s_{h}}{H} \in \mathbb{Q}$, then use Algorithm 1 with input $f$ and $h$ to find $g$ such that $f=g \circ h$. If such a $g$ exists, output $g$ and $h$ and terminate the algorithm; otherwise, go to next step. This step solves the case the left decomposition factor of $t$ being a non-trivial pol.

S5 Now $t$ does not have a pol decomposition in which the separant of the right decomposition factor equals $H$ and so the analysis in this section is correct. From (8), $H^{i}$ should be a factor of $t_{i}$, for $i=1, \ldots, d$. If there exists any $i$ such that $H^{i} \nmid t_{i}$, then output the empty set and terminate the algorithm.

S6 Let $c=t_{d} / H^{d}$. If $c \in \mathbb{Q}$, let $w=\frac{1}{a d} \frac{t_{d-1}}{H^{d-1}}$. By (10), output $p=H x_{o_{f}}+w$ and terminate the algorithm.
S7 If $c \notin \mathbb{Q}$, then by (8), $h$ is also a right decomposition factor of $c$. Let $t=c-c_{1}$, where $c_{1}$ denotes the constant term of $c$. Then $h$ is also a right decomposition factor of $t$. Go to S2.

EXAmPLE 3. $f=2 x_{2}\left(x_{2}^{2}+x_{1}\right) x_{3}+x_{2}^{4}+x_{2}^{3}+2 x_{1} x_{2}^{2}+x_{1} x_{2}+$ $x_{1}^{2}, H=x_{2}$. Let $t=f$, we have $d_{t}=1, t_{1}=2 x_{2}\left(x_{2}^{2}+x_{1}\right), H$ $t_{1}$. From $\mathbf{S 2}$ to $\mathbf{S 6}$, the algorithm does nothing. In $\mathbf{S 7}$, we get $t=2\left(x_{2}^{2}+x_{1}\right)$. Return to S2. In S3, $\frac{s_{t}}{H}=4 \in \mathbb{Q}$. By Algorithm 1 we find a $g=\frac{1}{4} x x_{1}+\frac{1}{4} x^{2}$ such that $f=$ $\left(\frac{1}{4} x x_{1}+\frac{1}{4} x^{2}\right) \circ\left(2 x_{2}^{2}+2 x_{1}\right)$.

\section{PSEUDO LINEAR CASE}

We will solve the following problem: for two given d-pols $H$ and $p$ with $d_{p}=1, s_{p}=H$, find a decomposition $p=r \circ h$ under the condition that $r$ is pseudo linear and $s_{h}=H$.

We write $d$-pols $p, r, h$ as the sum of total degree homogeneous parts:

$$
\begin{aligned}
& p=P_{d}+P_{d-1}+\ldots+P_{2}+P_{1} \\
& r=R_{d_{1}}+R_{d_{1}-1}+\ldots+R_{2}+R_{1} \\
& h=H_{d_{2}}+H_{d_{2}-1}+\ldots+H_{2}+H_{1}
\end{aligned}
$$

where $d, d_{1}, d_{2}$ denote the total degree of $p, r, h$ respectively. Notice that $p, r, h$ have no terms in $\mathbb{Q}$. Since $r$ is pseudo linear, $o_{R_{i}}<o_{R_{1}}$ for $2 \leq i \leq d_{1}$.

Denote $f f_{k}$ the sum of the monomials included in $f$ with total degree $k$. We assume that $d_{1}<d_{2}$ (the case $d_{1} \geq d_{2}$ is similar). Comparing the sum of the monomials with total degree $l(1 \leq l \leq d)$ in $p=r \circ h$, we have

$$
\begin{aligned}
P_{1} & =R_{1} \circ H_{1} \\
\cdots & \\
P_{k} & =R_{1} \circ H_{k}+R_{k} \circ H_{1}+\sum_{1<i<k} R_{i} \circ\left(\sum_{1 \leq j \leq k-1} H_{j}\right) \\
\ldots & \left(k<d_{1}\right) \\
P_{d_{1}} & \left.=R_{1} \circ H_{d_{1}}+R_{d_{1}} \circ H_{1}+\sum_{1<i<d_{1}} R_{1 \leq j \leq d_{1}-1} H_{j}\right) \\
\ldots & R_{d_{1}} \\
P_{s} & =R_{1} \circ H_{s}+\sum_{1<i \leq d_{1}} R_{1 \leq j \leq s-1} H_{j} \sum_{d_{2}}\left(d_{1}<s \leq d_{2}\right) \\
\ldots & =1 \sum_{v} R_{i} \circ\left(\sum_{1 \leq j \leq v} H_{j}\right) d_{v} \\
P_{v} & =R_{d_{1}} \circ H_{d_{2}}
\end{aligned}
$$

The basic idea of our algorithm is as follows: find $R_{1}, H_{1}$ from the first equation in (11) and substitute $R_{1}, H_{1}$ into the second equation to obtain $P_{2}^{\prime}=P_{2}-\left(R_{1} \circ H_{2}+R_{2} \circ H_{1}\right)$. From $P_{2}^{\prime}=0$, we obtain a system of linear equations in the coefficients of $R_{2}$ and $H_{2}$. To solve this linear equation system, we may obtain $R_{2}$ and $H_{2}$ and so on. To give a precise algorithm, we need to solve the following problems.

1. How to determine $d_{1}$ and $d_{2}$ ?

2. How can we obtain $R_{1}, H_{1}$ from the first equation of (11), that is, how to decompose linear $d$-pols with constant coefficients?

3. Do $R_{k}, H_{k}\left(1 \leq k \leq \min \left\{d_{1}, d_{2}\right\}\right)$ exist and are they unique? If they exist but are not unique, we will face the difficult problem of solving algebraic equations about the coefficients in the next step. 
4. If $d_{1}<d_{2}$, we will obtain $R_{i}, H_{i}\left(1 \leq i \leq d_{1}\right)$ firstly and then compute $H_{j}\left(d_{1}<j \leq d_{2}\right)$. We need to determine whether $H_{j}\left(d_{1}<j \leq d_{2}\right)$ is unique. Similarly, if $d_{1}>$ $d_{2}$, is $R_{j}\left(d_{2}<j \leq d_{1}\right)$ unique?

5. If $P_{1}=0$, what shall we do?

For the first problem, by Lemma 5 we have $d=d_{1} d_{2}$. It is obvious that $d_{2}=\operatorname{tdeg}(h) \geq \operatorname{tdeg}(H)+1(H$ is the separant of $h$ which has been given). So we will search all possible pairs $\left(d_{1}, d_{2}\right)$ satisfying these two conditions.

For the second problem, we have the following result.

LEMMA 7. Let $q=\sum_{k=0}^{n} a_{k} x_{k}, u=\sum_{i=0}^{m} b_{i} x_{i}$, $v=\sum_{j=0}^{n-m} c_{j} x_{j}$, where $m<n$ and $a_{k}, b_{i}, c_{j} \in \mathbb{Q}($ or $\mathbb{C})$. Then $q=u \circ v$ if and only if $\hat{q}=\hat{u} \hat{v}$, where $\hat{q}=\sum_{k=0}^{n} a_{k} y^{k}$, $\hat{u}=\sum_{i=0}^{m} b_{i} y^{i}, \hat{v}=\sum_{j=0}^{n-m} c_{j} y^{j}$.

Proof. From $q=u \circ v$, we have $q=\left(\sum_{i=0}^{m} b_{i} x_{i}\right) \circ\left(\sum_{j=0}^{n-m} c_{j} x_{j}\right)$ $=\sum_{i=0}^{m} \sum_{j=0}^{n-m} b_{i} c_{j} x_{i+j}=\sum_{k=0}^{n}\left(\sum_{i+j=k} b_{i} c_{j}\right) x_{k}$. Comparing the coefficients of $x_{i}$, we have $a_{k}=\sum_{i+j=k} b_{i} c_{j}$. Then $\hat{u} \hat{v}=\left(\sum_{i=0}^{m} b_{i} y^{i}\right)\left(\sum_{j=0}^{n-m} c_{j} y^{j}\right)=\sum_{k=0}^{n}\left(\sum_{i+j=k} b_{i} c_{j}\right) y^{k}=$ $\sum_{k=0}^{n} a_{k} y^{k}=\hat{q}$.

By Lemma 7, the problem of decomposing an LODE $q$ with constant coefficients is equivalent to the problem of factoring $\hat{q}$, so $R_{1}, H_{1}$ could have many choices. Since $o_{H} \leq$ $o_{h}$ and $r$ is pseudo linear, we have $o_{r}=o_{R_{1}}=o_{p}-o_{h} \leq$ $o_{p}-o_{H}$. So $o_{r}=o_{R_{1}} \leq \min \left\{o_{P_{1}}, o_{p}-o_{H}\right\}$. This may help us to reduce the choices of $R_{1}$ and $H_{1}$. Our algorithm will start with a possible pair $\left(R_{1}, H_{1}\right)$ and $\left(d_{1}, d_{2}\right)$ obtained from above.

Lemma 8. Let $q, u$ be total degree homogeneous $d$-pols. Let $\operatorname{ddeg}(q)=\tilde{d}, \operatorname{ddeg}(u)=\tilde{d}_{2}$, and $q_{\tilde{d}}, u_{\tilde{d}_{2}}$ denote the sum of the monomials in $q, u$ with differential degree $\tilde{d}, \tilde{d}_{2}$ respectively. $n$ is a positive integer, $a_{i} \in \mathbb{Q}(0 \leq i \leq n)$, we have:

(1). If $q=\left(a_{n} x_{n}+a_{n-1} x_{n-1}+\ldots+a_{1} x_{1}+a_{0} x\right) \circ u$, then $q_{\tilde{d}}=a_{n} x_{n} \circ u_{\tilde{d_{2}}}, \tilde{d}=n+\tilde{d}_{2}$.

(2). If $q=u \circ\left(a_{n} x_{n}+a_{n-1} x_{n-1}+\ldots+a_{1} x_{1}+a_{0} x\right)$, then $q_{\tilde{d}}=a_{n}^{t \operatorname{deg}(q)} u_{\tilde{d}_{2}} \circ x_{n}, \tilde{d}=n \cdot \operatorname{tdeg}(q)+\tilde{d}_{2}$.

Proof. Omitted. See the preprint [8].

Lemma 9. Let $q$ be a $d$-pol, $k=\min \left\{i: x_{i}\right.$ appears in $q\}, n$ a positive integer. If $q$ is not of the form $c x_{k}+q^{\prime}$, where $c \in \mathbb{Q}$ and $x_{k}$ does not appear in $q^{\prime}$, then $x_{k}$ appears in $x_{n} \circ q$.

Proof. Omitted. See the preprint [8].

The theorem below answers problem 3 affirmatively.

TheOREM 1. Let $R_{1}, H_{1}$ be linear d-pols and $P_{k}$ a $k$-total degree homogeneous $d$-pol with $k>1$. If there exist $k$-total degree homogeneous $d$-pols $R_{k}, H_{k}$ such that $o_{R_{k}}<o_{R_{1}}$ and $P_{k}=R_{1} \circ H_{k}+R_{k} \circ H_{1}$, then they are unique.

Proof. Suppose that $\left(R_{k 1}, H_{k 1}\right),\left(R_{k 2}, H_{k 2}\right)$ both satisfy the condition, that is

$$
P_{k}=R_{1} \circ H_{k 1}+R_{k 1} \circ H_{1}=R_{1} \circ H_{k 2}+R_{k 2} \circ H_{1}
$$

Since $R_{1}$ is linear, from (12) we have

$$
R_{1} \circ\left(H_{k 1}-H_{k 2}\right)+\left(R_{k 1}-R_{k 2}\right) \circ H_{1}=0
$$

Since $R_{k 1}-R_{k 2}$ and $H_{k 1}-H_{k 2}$ are still $k$-total degree homogeneous, we need only to prove that there exist no nonzero $R_{k}$ and $H_{k}$ such that $R_{1} \circ H_{k}=R_{k} \circ H_{1}$. Suppose such $H_{k}, R_{k}$ exist. Let $m=o_{R_{1}}, n=o_{H_{1}}$, and the sum of monomials included in $R_{k}, H_{k}$ with differential degree $\operatorname{ddeg}\left(R_{k}\right), \operatorname{ddeg}\left(H_{k}\right)$ be $\tilde{R}_{k}, \tilde{H}_{k}$ respectively. Compare the sum of monomials with maximal differential degree in both sides, since $R_{1}, H_{1}$ are linear, by Lemma 8 we have

$$
a_{1} x_{m} \circ \tilde{H}_{k}=b_{1}^{k} \tilde{R}_{k} \circ x_{n}
$$

where $a_{1}, b_{1}$ are the initial of $R_{1}, H_{1}$ respectively $\left(a_{1}, b_{1} \in\right.$ Q). Let $k=\min \left\{i: x_{i}\right.$ appears in $\left.\tilde{R}_{k} \circ x_{n}\right\}$. Then $k \geq n$, namely, $x_{i}(0 \leq i<n)$ does not appear in the right side of (13). By Lemma 9 , since $\tilde{H}_{k}$ is $k$-total degree homogeneous with $k>1$, we have $o_{\tilde{H}_{k}} \geq n$. Therefore,

$$
o_{H_{k}} \geq o_{\tilde{H}_{k}} \geq n=o_{H_{1}} .
$$

But by $R_{1} \circ H_{k}=R_{k} \circ H_{1}$, we have $o_{R_{1}}+o_{H_{k}}=o_{R_{k}}+$ $o_{H_{1}}$, which implies $o_{R_{1}} \leq o_{R_{k}}$. This contradicts with the hypothesis.

The fourth problem is easier to explain: when $d_{1} \geq d_{2}$, we obtain all $H^{\prime} s$ firstly, then we have obtained the right decomposition factor of $p$ and the corresponding left decomposition factor is unique certainly; when $d_{1}<d_{2}$, we obtain all $R^{\prime} s$ firstly. $H_{i}\left(d_{1}<i \leq d_{2}\right)$ is unique from the fact: for two given $d$-pols $f$ and $g$, if $g$ is linear, then the $h$ which satisfies $f=g \circ h$ is unique if it exists.

For the last problem, the method mentioned above is also valid. Let $k=\min \left\{i: P_{i} \neq 0\right\}$. We will obtain $R_{1}, H_{k}$ from the relation $R_{1} \circ H_{k}=P_{k}$ and get other $R_{i}, H_{j}$ by solving linear equations with their coefficients as unknowns. When $R_{1}$ is given, the uniqueness for $\left.H_{j}(k \nmid j)\right)$ is obvious and the uniqueness for $H_{j}(k \mid j)$ is guaranteed by Theorem 2 which follows below.

Lemma 10. If $x_{1} \circ r=s \circ t$, where $r, s, t$ are total degree homogeneous and $\operatorname{tdeg}(s)>1$, then there exists a d-pol $s^{\prime}$ such that $s=x_{1} \circ s^{\prime}$.

Proof. Omitted. See the preprint [8].

TheOREM 2. Let $R_{1}, H_{1}$ be linear $d$-pols and $P_{i k} a i \cdot k$ total degree homogeneous $d$-pol. If there exist an $i$-total degree homogeneous $d$-pol $R_{i}$ and $i \cdot k$-total degree homogeneous $d$-pol $H_{i k}$ such that $o_{R_{i}}<o_{R_{1}}$ and $P_{i k}=R_{1} \circ H_{i k}+R_{i} \circ H_{k}$, then they are unique.

Proof. Since $R_{1}, H_{1}$ are linear, we need only to prove that there exist no nonzero $H_{i k}$ and $R_{i}$ such that $R_{1} \circ H_{i k}=$ $R_{i} \circ H_{k}$, as in Theorem 1. Suppose that such $H_{i k}, R_{i}$ exist. Let $m=o_{R_{1}}$, and the sum of the monomials included in $H_{i k}, R_{i}, H_{k}$ with differential degree $\operatorname{ddeg}\left(H_{i k}\right), \operatorname{ddeg}\left(R_{i}\right)$, $\operatorname{ddeg}\left(H_{k}\right)$ be $\tilde{H}_{i k}, \tilde{R}_{i}, \tilde{H}_{k}$ respectively. Compare the sum of monomials with maximal differential degree in both sides we have

$$
a_{1} x_{m} \circ \tilde{H}_{i k}=\tilde{R}_{i} \circ \tilde{H}_{k}
$$

where $a_{1}$ is the initial of $R_{1}\left(a_{1} \in \mathbb{Q}\right)$. By Lemma 10, there exists some differential polynomial $R_{i}^{\prime}$ such that $\tilde{R}_{i}=x_{m}$ 。 $R_{i}^{\prime}$, so we have $o_{R_{1}}=m \leq o_{\tilde{R}_{i}} \leq o_{R_{i}}$, which contradicts with the hypothesis.

To get $R_{1}$ and $H_{k}$, we need to solve the problem: given a total degree homogeneous d-pol $q$, how to obtain $d$-pols $s, t$ such that $q=s \circ t$ and $s$ is linear. 
Assume that $q$ can be decomposed as $q=s \circ t$, where $s$ is linear and $t$ is total degree homogeneous. By Lemma 7, we can assume that $s=x_{1}+a x, a \in \mathbb{C}$. Let $\tilde{d}=\operatorname{ddeg}(q)$. Since $\operatorname{ddeg}(q)-\operatorname{ddeg}(t)=1$, we write $q$ and $t$ as differential degree homogeneous parts:

$$
\begin{aligned}
q & =Q_{\tilde{d}}+Q_{\tilde{d}-1}+\ldots+Q_{1}+Q_{0} \\
t & =T_{\tilde{d}-1}+T_{\tilde{d}-2}+\ldots+T_{1}+T_{0}
\end{aligned}
$$

Compare the sum of the monomials with differential degree $i(0 \leq i \leq \tilde{d})$ of both sides of $q=s \circ t$, we have

$$
Q_{\tilde{d}}=x_{1} \circ T_{\tilde{d}-1}, \ldots, Q_{0}=a x \circ T_{0} .
$$

So we have: $T_{0}=\frac{Q_{0}}{a}, \quad T_{i}=\frac{Q_{i}-x_{1} \circ T_{i-1}}{a}(1 \leq i \leq \tilde{d}-1)$. Substituting $T_{0}, \ldots, T_{\tilde{d}-1}$ into $Q_{\tilde{d}}-x_{1} \circ T_{\tilde{d}-1}=0$, we have

$$
a^{\tilde{d}} Q_{\tilde{d}}-a^{\tilde{d}-1}\left(x_{1} \circ Q_{\tilde{d}-1}\right)+\ldots+(-1)^{\tilde{d}} x_{\tilde{d}} \circ Q_{0}=0 .
$$

When $q$ is given, the $Q^{\prime} s$ are decided. So we obtain a set of equations about $a$. Conversely, if $a$ satisfies these equations and $a \neq 0$, then $s=x_{1}+a x$ is a left decomposition factor of $q$ (to deal with the case $a=0$, we will use Algorithm 2). When consider $s$ in $\mathbb{Q}\{x\}$, we may use Lemma 7 to obtain a linear left decomposition factor of maximal order of $q$.

Algorithm 5. Input: a total degree homogeneous d-pol $q$.

Output: the linear left decomposition factor $s$ of $q$ with maximal order, if it exists.

S1 Using Algorithm 2 to get the maximal $n$ such that $q=$ $x_{n} \circ q^{\prime}$. Let $\tilde{d}=\operatorname{ddeg}\left(q^{\prime}\right)$, write $q^{\prime}$ as $q^{\prime}=Q_{\tilde{d}}+Q_{\tilde{d}-1}+$ $\ldots+Q_{1}+Q_{0}$, where $Q_{i}$ is the sum of the monomials included in $q^{\prime}$ with differential degree $i(0 \leq i \leq \tilde{d})$. Calculate $V_{i}=x_{\tilde{d}-i} \circ Q_{i}(0 \leq i \leq \tilde{d})$.

S2 Let $w=a^{\tilde{d}} V_{\tilde{d}}-a^{\tilde{d}-1} V_{\tilde{d}-1}+\ldots+(-1)^{\tilde{d}-i} a^{i} V_{i}+\ldots+$ $(-1)^{\tilde{d}-1} a V_{1}+(-1)^{\tilde{d}} V_{0}$, and $S$ the set of the coefficients of each monomial included in $w$. We obtain the equations $S=0$ of $a$.

S3 To solve the equations $S=0$ in $\mathbb{Q}$ with $a \neq 0$, we basically need to compute the GCD $g$ of the pols in $S$. If $g \in \mathbb{Q}$, then there is no solution for $a$ and output $s=$ $x_{n}$ (if $n=0$, then the decomposition does not exist). Otherwise, let $g=a^{l}\left(a^{m}+a_{m-1} a^{m-1}+\ldots+a_{1} a+a_{0}\right)$ where $a_{i} \in \mathbb{Q}$ and $a_{0} \neq 0$. By Lemma 7 , the output is $s=x_{n} \circ\left(x_{m}+a_{m-1} x_{m-1}+\ldots+a_{1} x_{1}+a_{0} x\right)$.

EXAMPLE 4. Let $q=2 x_{1} x_{3}+2 x_{2}^{2}+x x_{3}+3 x_{1} x_{2}+2 x x_{2}+$ $x_{1}^{2}-x x_{1}-x^{2}$. In $S 1, n=0$ and we have $\tilde{d}=4, Q_{4}=2 x_{1} x_{3}+$ $2 x_{2}^{2}, Q_{3}=x x_{3}+3 x_{1} x_{2}, Q_{2}=2 x x_{2}+x_{1}^{2}, Q_{1}=-x x_{1}, Q_{0}=$ $-x^{2}$. So,

$$
\left\{\begin{array}{l}
V_{4}=Q_{4}=2 x_{1} x_{3}+2 x_{2}^{2} \\
V_{3}=x_{1} \circ Q_{3}=x x_{4}+4 x_{1} x_{3}+3 x_{2}^{2} \\
V_{2}=x_{2} \circ Q_{2}=2 x x_{4}+6 x_{1} x_{3}+4 x_{2}^{2} \\
V_{1}=x_{3} \circ Q_{1}=-\left(x x_{4}+4 x_{1} x_{3}+3 x_{2}^{2}\right) \\
V_{0}=x_{4} \circ Q_{0}=-\left(8 x_{1} x_{3}+6 x_{2}^{2}+2 x x_{4}\right)
\end{array}\right.
$$

In S2, we have $p=a^{4} V_{4}-a^{3} V_{3}+a^{2} V_{2}-a V_{1}+V_{0}=$ $\left(2 a^{4}-4 a^{3}+6 a^{2}+4 a-8\right) x_{1} x_{3}+\left(2 a^{4}-3 a^{3}+4 a^{2}+3 a-\right.$ 6) $x_{2}^{2}+\left(-a^{3}+2 a^{2}+a-2\right) x x_{4}=0$. Collecting the coefficients, we have

$$
\left\{\begin{array}{l}
h_{1}=2 a^{4}-4 a^{3}+6 a^{2}+4 a-8 \\
h_{2}=2 a^{4}-3 a^{3}+4 a^{2}+3 a-6 \\
h_{3}=-a^{3}+2 a^{2}+a-2
\end{array}\right.
$$

In S3, compute the GCD of $h_{1}, h_{2}, h_{3}$, we have $g=a^{2}-1$. So the left decomposition factor of $q$ with the maximal order is: $s=x_{2}-x$.

We can now present the algorithm.

Algorithm 6. Input: $d$-pols $f, p, H$ with $d_{p}=1, i_{p}=H$. Output: d-pols $g, h$ such that $f=g \circ h$ where $h$ is a right decomposition factor of $p$ and $\frac{s_{h}}{H} \in \mathbb{Q}$. If such $g$ and $h d o$ not exist, return nothing.

S1 Let $d=\operatorname{tdeg}(p)$. Write $p$ as $p=\sum_{i=1}^{d} P_{i}$ where $P_{i}$ denotes the sum of the monomials in $p$ with total degree $i(1 \leq i \leq d)$

S2 Let $k=\min \left\{i: P_{i} \neq 0\right\}$. If $k=1$, let $L=P_{1}$; else, let $L$ be the linear left decomposition factor of $P_{k}$ with maximal order obtained with Algorithm 5. If $L$ does not exist, the algorithm terminates; otherwise, let $S=\left\{\left(A, d^{\prime}\right): A\right.$ is a linear left decomposition factor of $L$ and $i_{A}=1, d^{\prime}$ is an integer such that $d^{\prime} \mid \operatorname{tdeg}(p)$ and $\left.d^{\prime} \geq \operatorname{tdeg}(H)+1\right\}$. By Lemma 7, a left decomposition factor of $L$ can be found by factoring a univariate pol.

S3 If $S \neq \emptyset$, select a $\left(A, d^{\prime}\right)$ from $S$ and let $R_{1}=A, d_{2}=$ $d^{\prime}, d_{1}=\operatorname{tdeg}(p) / d^{\prime}$. Otherwise, the algorithm terminates.

$\mathbf{S} 4$ Let $H_{i}=0(1 \leq i<k)$. Solve the equations (11) to find $H_{k}, R_{2}, H_{k+1}, \ldots, R_{d_{1}}, H_{d_{2}}$ by treating the coefficients of $R^{\prime} s$ and $H^{\prime} s$ as unknowns with the conditions $o_{R_{l}}<$ $o_{R_{1}}, o_{H_{j}} \leq o_{p}-o_{R_{1}}$ and $\operatorname{tdeg}\left(R_{l}\right)=l, \operatorname{tdeg}\left(H_{j}\right)=j$ for $2 \leq l \leq d_{1}, k \leq j \leq d_{2}$. By Theorems 1 and 2 , such a solution must be unique if it exists. If the solution does not exist, $S:=S-\left\{\left(A, d^{\prime}\right)\right\}$, go to S3. Otherwise, let $h=H_{k}+H_{k+1}+\ldots+H_{d_{2}}$.

S5 if $\frac{s_{h}}{H} \notin \mathbb{Q}, S:=S-\left\{\left(A, d^{\prime}\right)\right\}$, go to S3.

S6 Execute Algorithm 1 with input $f, h$, if we obtain a $g$ such that $f=g \circ h$, then output $g$ and $h$; else, $S:=$ $S-\left\{\left(A, d^{\prime}\right)\right\}$, go to S3.

The example follows below shows how to obtain the decomposition of a $d$-pol $p$ when we have known that the left decomposition factor of $p$ is pseudo linear.

EXAMPLE 5. Let $p=\left(x x_{2}+x_{1}^{2}\right)^{2}+4 x_{1}\left(x x_{2}+x_{1}^{2}\right)+4 x_{1}^{2}-$ $x x_{1}+3 x_{1} x_{2}+x x_{3}+2 x_{2}-2 x$.

S1. $d=\operatorname{tdeg}(p)=4$, write $p$ as $p=P_{4}+P_{3}+P_{2}+P_{1}$, where $P_{4}=x^{2} x_{2}^{2}+x_{1}^{4}+2 x x_{1}^{2} x_{2}, P_{3}=4 x x_{1} x_{2}+4 x_{1}^{3}, P_{2}=$ $4 x_{1}^{2}-x x_{1}+3 x_{1} x_{2}+x x_{3}, P_{1}=2 x_{2}-2 x$.

S2. From $P_{1}=2\left(x_{2}-x\right)=R_{1} \circ H_{1}$ and by Lemma 7 , we choose $R_{1}$ to be one of $x_{2}-x, x_{1}+x, x_{1}-x$. Since $\operatorname{tdeg}(p)=4, \operatorname{tdeg}(H)=2$, we have $S=\left\{\left(x_{2}-x, 2\right),\left(x_{2}-\right.\right.$ $\left.x, 4),\left(x_{1}+x, 2\right),\left(x_{1}+x, 4\right),\left(x_{1}-x, 2\right),\left(x_{1}-x, 4\right)\right\}$.

S3. For $R_{1}=x_{2}-x, d_{2}=2, H_{1}=2 x, d_{1}=d / d_{2}=2$. Substituting these into (11), we have:

$$
\begin{aligned}
P_{2} & =\left(x_{2}-x\right) \circ H_{2}+R_{2} \circ 2 x \\
P_{3} & =R_{2} \circ\left(H_{1}+H_{2}\right) \\
P_{4} & =R_{2} \circ H_{2}
\end{aligned}
$$


S4. Since $o_{R_{2}}<2, o_{H_{2}} \leq o_{f}-o_{R_{1}}=3-2=1$ and $R_{2}, H_{2}$ are 2-total degree homogeneous, so we can assume

$$
R_{2}=a_{1} x^{2}+a_{2} x x_{1}+a_{3} x_{1}^{2}, H_{2}=b_{1} x^{2}+b_{2} x x_{1}+b_{3} x_{1}^{2}
$$

Substitute them in (14), we have: $2 b_{1} x x_{2}+\left(2 b_{1}-b_{3}+4 a_{3}-\right.$ 4) $x_{1}^{2}+\left(b_{2}-1\right) x x_{3}+\left(3 b_{2}-3\right) x_{1} x_{2}+2 b_{3} x_{1} x_{3}+2 b_{3} x_{2}^{2}+\left(4 a_{2}-\right.$ $\left.b_{2}+1\right) x x_{1}+4 a_{1} x^{2}=0$. We obtain the equations for the coefficients of $\mathrm{R}_{2}, \mathrm{H}_{2}$ :

$$
\left\{\begin{array}{l}
2 b_{1}=2 b_{3}=a_{1}=0,2 b_{1}-b_{3}+4 a_{3}-4=0 \\
b_{2}-1=0,3 b_{2}-3=0,4 a_{2}-b_{2}+1=0
\end{array}\right.
$$

The unique solution is: $a_{1}=0, a_{2}=0, a_{3}=1 ; b_{1}=0, b_{2}=$ $1, b_{3}=0$. So we obtain $R_{2}=x_{1}^{2}, H_{2}=x x_{1}$. Now we substitute $R_{1}, R_{2}, H_{1}, H_{2}$ in (15) and (16), we find that they really hold. So we have the decomposition for $p$ :

$$
p=\left(R_{1}+R_{2}\right) \circ\left(H_{1}+H_{2}\right)=\left(x_{1}^{2}+x_{2}-x\right) \circ\left(x x_{1}+2 x\right) .
$$

When we choose the other elements of $S$, we do not obtain solutions for $\mathrm{R}_{2}, \mathrm{H}_{2}$. Here we omit the calculation.

\section{THE GENERAL CASE AND EXPERIMEN- TAL RESULTS}

Algorithm 7. Input: a $f$ in $\mathbb{Q}\{x\}$.

Output: a non-trivial decomposition $f=g \circ h$, if such $g, h$ exist.

S1 Find a pol $g \in \mathbb{Q}[x]$ and an $h \in \mathbb{Q}\{x\}$ such that $f=g \circ h$ with Algorithm 3. If such $g$ and $h$ exist, output $g, h$. Otherwise, go to next step.

S2 Let $d=d_{f}$, write $f$ as the form $f_{d} x_{o_{f}}^{d}+f_{d-1} x_{o_{f}}^{d-1}+$ $\ldots+f_{1} x_{o_{f}}+f_{0}$, where $f_{i}$ denotes the coefficient of $x_{o_{f}}^{i}$ $(0 \leq i \leq d)$. Let $T=\left\{H: H=1\right.$ or $H^{i}$ is a factor of $\left.f_{i}(1 \leq i \leq d)\right\}$. To make the selection of factors unique, we assume that $H$ is with integer coefficients and primitive.

S3 If $T \neq \phi$, choose $H \in T$, go to next step; otherwise, terminate the algorithm and return "no non-trivial decomposition exists".

S4 By (8), $H$ could be the separant of $h$. Execute Algorithm 4 with input $f, H$. There are three cases:

(1) We obtain a decomposition of $f$. Output the decomposition and terminate the algorithm.

(2) We obtain a $d$-pol $p$ such that if $h$ is a right decomposition factor of $f$ with separant $H$, then $h$ is a right decomposition factor of $p$ and the corresponding left decomposition factor of $p$ w.r.t. $h$ is pseudo linear. Go to next step.

(3) The output is the empty set. Let $T:=T-\{H\}$, go to S3;

S5 Execute Algorithm 6 with input $f, p, H$. If we get a decomposition $f=g \circ h$, then output $g$ and $h$; otherwise $T:=T-\{H\}$, go to $\mathbf{S 3}$.

EXAMPLE 6. Let $f=2 x_{1} x_{3}+4 x_{1}^{2} x_{2}^{2}+4 x_{1}^{2} x_{2}+4 x_{1}^{3} x_{2}+$ $4 x_{1} x_{2} x+x_{1}^{2}+2 x_{1}^{3}+2 x_{1} x+x_{1}^{4}+2 x_{1}^{2} x+x^{2}+2 x_{2}^{2}+x_{2}+$ $2 x_{1} x_{2}+x_{1}$.

S1. Execute Algorithm 3, we find that $f$ does not have a pol decomposition.

\begin{tabular}{|c|c|c|c|}
\hline$\left(o_{f}, t_{f}, l_{f}\right)$ & time $(\mathrm{s})$ & $\left(o_{f}, t_{f}, l_{f}\right)$ & time $(\mathrm{s})$ \\
\hline$(2,10,32)$ & 0.237 & $(2,20,116)$ & 1.174 \\
\hline$(2,30,254)$ & 4.200 & $(2,40,414)$ & 12.938 \\
\hline$(2,50,624)$ & 35.021 & $(3,10,102)$ & 2.365 \\
\hline$(3,12,322)$ & 14.078 & $(3,15,368)$ & 11.167 \\
\hline$(4,8,415)$ & 19.786 & $(4,10,555)$ & 32.171 \\
\hline$(5,8,1084)$ & 55.986 & $(6,6,596)$ & 68.843 \\
\hline$(7,6,1308)$ & 164.44. & $(8,5,325)$ & 14.266 \\
\hline$(9,4,415)$ & 19.786 & $(10,4,677)$ & 72.534 \\
\hline
\end{tabular}

Table 1: Decomposing Randomly Generated Differential Polynomials

\begin{tabular}{|c|c|c|c|}
\hline$\left(o_{h}, t_{g}\right)$ & $\left(o_{h}, t_{h}\right)$ & $\left(o_{f}, t_{f}, l_{f}\right)$ & time $(\mathrm{s})$ \\
\hline$(0,8)$ & $(1,8)$ & $(1,64,639)$ & 10.079 \\
\hline$(1,6)$ & $(1,8)$ & $(2,48,1174)$ & 85.640 \\
\hline$(1,4)$ & $(2,4)$ & $(3,16,458)$ & 38.672 \\
\hline$(1,4)$ & $(3,2)$ & $(4,8,994)$ & 95.063 \\
\hline$(1,4)$ & $(3,4)$ & $(4,16,970)$ & 144.467 \\
\hline$(2,4)$ & $(1,4)$ & $(3,16,1229)$ & 189.109 \\
\hline$(2,3)$ & $(2,4)$ & $(4,12,1360)$ & 120.093 \\
\hline$(2,3)$ & $(3,2)$ & $(5,6,709)$ & 90.405 \\
\hline$(3,2)$ & $(1,4)$ & $(4,8,231)$ & 15.562 \\
\hline$(3,2)$ & $(2,4)$ & $(5,8,535)$ & 32.891 \\
\hline
\end{tabular}

Table 2: Decomposing Differential Polynomials Composed Randomly

S2. $d=d_{f}=1, f_{1}=2 x_{1}, T=\left\{1, x_{1}\right\}$.

S3. Select $H=1$.

S4. Execute Algorithm 4, we obtain $p=x_{1}$.

S5. By Algorithm 6, we get no decomposition for $f$.

Now, return to $S 3$. Select $H=x_{1}$.

S4. Execute Algorithm 4, we obtain $p=2 x_{1} x_{3}+4 x_{1}^{2} x_{2}^{2}+$ $4 x_{1}^{2} x_{2}+4 x_{1}^{3} x_{2}+4 x_{1} x_{2} x+x_{1}^{2}+2 x_{1}^{3}+2 x_{1} x+x_{1}^{4}+2 x_{1}^{2} x+$ $x^{2}+2 x_{2}^{2}+x_{2}+2 x_{1} x_{2}+x_{1}$.

S5. By Algorithm 6, we have $g=x_{1}+x^{2}, h=2 x_{1} x_{2}+$ $x_{1}+x_{1}^{2}+x$. So we obtain a decomposition $f=g \circ h$.

We may use Algorithm 7 recursively to find an irreducible decomposition $f=\left(x_{1}+x^{2}\right) \circ\left(x_{1}+x\right) \circ\left(x_{1}^{2}+x\right)$. So the solution of $f=0$ is reduced to the solving of three first order ODEs.

\subsection{Experimental Results}

We implement Algorithm 7 in Maple. In Table 1, we generate a $d$-pol randomly and decompose it. All the $d$-pols in Table 1 are indecomposable. In Table 2, we generate two $d$-pols $g$ and $h$ randomly and decompose $f=g \circ h$. These $g$ and $h$ could be found in [8]. The running times are collected on a PC with a $1.6 \mathrm{G}$ CPU and $128 \mathrm{M}$ memory and are given in seconds. In the table, $t_{f}$ means the total degree of $f$ and $l_{f}$ means the number of terms in $f$. From these results, we may conclude that our algorithm is quite efficient in handling large $d$-pols with hundreds of terms.

\subsection{Rational Function Coefficient Case}

If the coefficient field is $\mathbb{Q}(t)$, Algorithm 3, Lemma 7, and Algorithm 5 are not correct anymore. For the pol decomposition of $d$-pols, we have given an algorithm [8]. Corresponding to Lemma 7 , we need to decompose linear $d$-pols 
in $\mathbb{Q}(t)\{x\}$, which is much more difficult than the constant coefficient case and has been solved in [4, 26, 18, 23]. Corresponding to Algorithm 5, to find linear left decomposition factors of a total degree homogeneous $d$-pol with coefficients in $\mathbb{Q}(t)$, we need to solve high degree and high order differential equations. How to give an efficient algorithm to find such a left decomposition factor needs further research. While the uniqueness in Theorem 1 and Theorem 2 is still correct, which implies that the method in this paper is also valid in many cases for the decomposition of $f$ in $\mathbb{Q}(t)\{x\}$, such as when the given $d$-pol $f$ is pseudo linear.

\section{CONCLUSION}

We give an efficient algorithm for decomposing $d$-pols in one variable and with constant coefficients. Besides arithmetic operations, the algorithm needs only factorization of multi-variable pols and solution of linear equation systems. Experimental results show that this algorithm can be used to decompose large $d$-pols.

Many problems on the decomposition of $d$-pols are still open. The decomposition of pols is unique in certain sense [17]. Similar results were proved for Ore polynomials and hence for LODEs [20]. It is interesting to see whether this property is correct for $d$-pol decomposition. Whether we can extend the methods in $[16,9]$ to give a pol time decomposition algorithm for $d$-pols is also interesting. Finally, to give an efficient algorithm to decompose $d$-pols with coefficients of rational functions is also desirable.

Acknowledgment. We would like to thank the anonymous referees for providing valuable suggestions on this paper.

\section{REFERENCES}

[1] V.S. Alagar and M. Thanh. Fast decomposition algorithms. In Proc. EUROCAL 85, vol 2, 150-153, Springer, 1985.

[2] M.A. Barkatou and E. Pflügel. On the equivalence problem of linear differential systems and its application for factoring completely reducible systems. In Proc. of ISSAC'98, 268-275, ACM Press, 1998.

[3] D.R. Barton and R.E. Zippel. Polynomial decomposition algorithms. J. of Symbolic Computation, 1(2), 159-168, 1985.

[4] M. Bronstein. An improved algorithm for factorizing linear ordinary differential operators. In Proc. of ISSAC'94, ACM Press, 336-340, 1994.

[5] M. Bronstein and M. Petkovšek. On Ore rings, linear operators and factorization. Programming $\mathcal{E}$ Computer Software 20, 1, 27-44, 1994.

[6] T. Cluzeau. Factorization of differential systems in characteristic $p$. In Proc. ISSAC'03, 58-65, ACM Press, 2003.

[7] A. Fredet. Factorization of linear differential operators in exponential extensions. In Proc. of ISSAC03, 103-110, ACM Press, 2003.

[8] X.S. Gao and M. Zhang. Decomposition of differential polynomials. In MM Res. Preprints, No. 22, 163-185, 2003. http://www.mmrc.iss.ac.cn/pub/mm-pre.html

[9] J.von zur Gathen. Functional decomposition of polynomials: the wild case. J. of Symbolic Computation, 9, 437-452, 1990; the tame case. J. of Symbolic Computation, 9, 281-299, 1990.
[10] M. Giesbrecht and Y. Zhang. Factoring and decomposing Ore polynomials over $F q(t)$. In Proc. of ISSAC03, 127-135, ACM Press, 2003.

[11] D.Y. Grigor'ev. Complexity of factoring and calculating the GCD of linear ordinary differential operators. J. of Symbolic Computation, 10, 7-37, 1990.

[12] J. Gutierrez, T. Recio and C.R. de Velasco. Polynomial decomposition of almost quadratic complexity. In Proc. AAECC 6, Springer, 1989.

[13] Z. Li, F. Schwarz and S.P. Tsarev. Factoring systems of linear PDEs with finite-dimensional solution spaces. J. Symbolic Computation 36(3-4), 443-471, 2003.

[14] E.R. Kolchin. Differential Algebra and Algebraic Groups, Academic Press, London, 1973.

[15] L. Königsberger. Allgemeine Untersuchungen aus der Theorie der Differentialgleichungen, Teubner, Leipzig, 1882 .

[16] D. Kozen and S. Landau. Polynomial decomposition algorithms. J. of Symbolic computation 7, 445-456, 1989.

[17] J.F. Ritt. Prime and composite pols. Trans. AMS, 23, 51-66, 1922.

[18] F. Schwarz. A factoring algorithm for linear ordinary differential equations. In Proc. of ISSAC 89, ACM Press, 17-25, 1989.

[19] M.F. Singer. Liouillian solutions of $n$th order homogeneous linear differential equations. Amer. J. of Math., 103(4), 661-682, 1981.

[20] O. Ore. Theory of non-commutative polynomials. The Annals of Mathematics, 34(3), 480-508, 1933.

[21] M.F. Singer and F. Ulmer. Galois groups of second and third order linear differential equations. J. of Symbolic Computation 16, 9-36, 1993.

[22] M. Sosnin. Decomposition of polynomial ordinary differential equations. Krasnoyarsk, to be published, 1999.

[23] S.P. Tsarev. An algorithm for complete enumeration of all factorizations of a linear ordinary differential operator. In Proc. of ISSAC'96, 226-231, ACM Press, 1996.

[24] S.P. Tsarev. On factorization of non-linear ordinary differential equations. In Proc. ISSAC 99, 159-164, ACM Press, 1999.

[25] H. Umemura. On the irreducibility of the first differential equation of Painleve. In Algebraic geometry and commutative algebra in honor of Masayoshi Nagata, Tokyo, 101-109, 1987.

[26] M. van Hoeij. Factorization of differential operators with rational functions coefficients. J. Symbolic Computation 24(5), 537-561, 1997.

[27] M. van Hoeij. Formal solutions and factorization of differential operators with power series coefficients. $J$. Symbolic Computation, 24, 1-30 1997.

[28] M. Van der Put and M.F. Singer. Galois theory of linear differential equations, Springer, Berlin, 2003.

[29] R.E. Zippel. Rational function decomposition. In Proc. of ISSAC'91, 1-6, ACM Press, 1991. 